\title{
Strong spin-dependent negative differential resistance in composite graphene superlattices
}

\author{
J. Munárriz, ${ }^{1}$ C. Gaul, ${ }^{1,2,3}$ A. V. Malyshev, ${ }^{1,4}$ P. A. Orellana, ${ }^{5}$ C. A. Müller, ${ }^{6,7}$ and F. Domínguez-Adame ${ }^{1}$ \\ ${ }^{1}$ GISC, Departamento de Física de Materiales, Universidad Complutense, E-28040 Madrid, Spain \\ ${ }^{2}$ CEI Campus Moncloa, UCM-UPM, Madrid, Spain \\ ${ }^{3}$ Max Planck Institute for the Physics of Complex Systems, 01187 Dresden, Germany \\ ${ }^{4}$ Ioffe Physical-Technical Institute, 26 Politechnicheskaya Street, 194021 St. Petersburg, Russia \\ ${ }^{5}$ Departamento de Física, Universidad Técnica Federico Santa María, Casilla 110 V, Valparaíso, Chile \\ ${ }^{6}$ Centre for Quantum Technologies, National University of Singapore, Singapore 117543, Singapore \\ ${ }^{7}$ Department of Physics, University of Konstanz, 78457 Konstanz, Germany
}

(Received 21 November 2012; published 17 October 2013)

\begin{abstract}
We find clear signatures of spin-dependent negative differential resistance in compound systems comprising a graphene nanoribbon and a set of ferromagnetic insulator strips deposited on top of it. The periodic array of ferromagnetic strips induces a proximity exchange splitting of the electronic states in graphene, resulting in the appearance of a superlattice with a spin-dependent energy spectrum. The electric current through the device can be highly polarized and both the current and its polarization manifest nonmonotonic dependence on the bias voltage. The device operates therefore as an Esaki spin diode, which opens possibilities to design new spintronic circuits.
\end{abstract}

DOI: 10.1103/PhysRevB.88.155423

PACS number(s): 72.80.Vp, 72.20.Ht, 85.75.Mm

\section{INTRODUCTION}

Since the pioneering work by Esaki, ${ }^{1}$ quantum tunneling and negative differential resistance (NDR) have been the underlying principle of operation of various quantum devices. $^{2-4}$ NDR is often related to the resonant tunneling of carriers; when the chemical potential of a lead approaches one of the resonant levels of a device, the current $I$ increases. However, the resonant level position can depend on the applied voltage $V$, which can finally drive the system out of resonance. Then, the current decreases dramatically with a further increase of the voltage. The resulting $I-V$ characteristics are typically $\mathrm{N}$-shaped and include a region with NDR. Such a conductance anomaly can, for example, be observed in semiconductor heterostructures, ${ }^{2}$ semiconductor superlattices, ${ }^{5}$ conductor/superconductor junctions, ${ }^{6}$ carbon nanotubes, ${ }^{7}$ molecular systems, ${ }^{4}$ and at the atomic scale. ${ }^{8}$

Due to its remarkable charge transport properties ${ }^{9}$ and long spin-coherence length, ${ }^{10-14}$ graphene is a very promising material for spintronics. ${ }^{15,16}$ Graphene nanoribbons (GNRs) with tailored edges (zigzag or armchair) provide means to generate and manipulate spin-polarized electrons. ${ }^{17}$ In this regard, signatures of NDR for spin-down electrons in Bedoped zigzag GNRs have already been found by Wu et al., ${ }^{18}$ where spin-polarized edge states play an important role.

Here, we consider a spin-dependent superlattice realized by ferromagnetic insulator strips ${ }^{19}$ deposited on top of an armchair GNR. Similar proposals on (ferromagnetic) superlattices of graphene have been presented recently. Yu et al. ${ }^{20}$ have studied a superlattice realized by stubs in the shape of a zigzag GNR with a ferromagnetic insulator on top of the whole system. They found strongly spin-dependent minibands and minigaps, but they did not study the effect of a bias voltage, nor have they found NDR. Niu et al. ${ }^{21}$ and Faizabadi et al. ${ }^{22}$ have investigated a superlattice made of gated ferromagnetic strips on top of graphene. However, the finite width of the GNR and the quantization of the transverse momentum was not taken into account. Instead, they took the incident angle as a free parameter. They found that spin polarization of tunneling conductance and magnetoresistance exhibit oscillatory behavior as a function of the gate voltage, but they did not consider the bias voltage either. Finally, Ferreira et al. $^{23}$ studied an armchair GNR under a spinindependent superlattice and a bias field, which leads to a spin-independent NDR effect.

In this paper we propose a graphene-based device whose $I-V$ characteristics show spin-dependent NDR with high peak-to-valley ratios, which could be an important building block for future spintronic devices. The structure of the paper is as follows. In Sec. II we present the setup of a gapped armchair GNR with several strips of a ferromagnetic insulator on top of it, which creates a spin-dependent superlattice. We compute the stationary wave function across the sample and the transmission coefficient for a given spin, energy, and bias voltage. The resulting current-voltage characteristics of the device, comprising a spin-filtering effect and a strong spin-dependent NDR, are discussed in the subsequent Sec. III, while Sec. IV concludes the paper and provides an outlook on possible further developments.

\section{SETUP AND FORMALISM}

The proposed system is composed of a rectangular GNR of width $W \simeq 9.8 \mathrm{~nm}$, connected to source and drain leads, and $N=5$ rectangular strips of a ferromagnetic insulator arranged periodically on top of the GNR (see the upper panel of Fig. 1). As we discuss later, this number of ferromagnetic strips is enough to reveal clear signatures of spin-dependent NDR. The width of the strips is $a=23.9 \mathrm{~nm}$ and the spacing between them is $b=55.8 \mathrm{~nm}$. It is known that both the width and the edge type of a GNR strongly affect its electronic properties. Experimental evidences ${ }^{24}$ and $a b$ initio calculations ${ }^{25}$ show that the spectrum of a GNR with armchair edges has a gap, which is inversely proportional to the width $W$ and depends on the remainder $\left(2 W / a_{0} \bmod 3\right)$, where $a_{0}=0.246 \mathrm{~nm}$ is the lattice constant, i.e., the width of the graphene lattice 


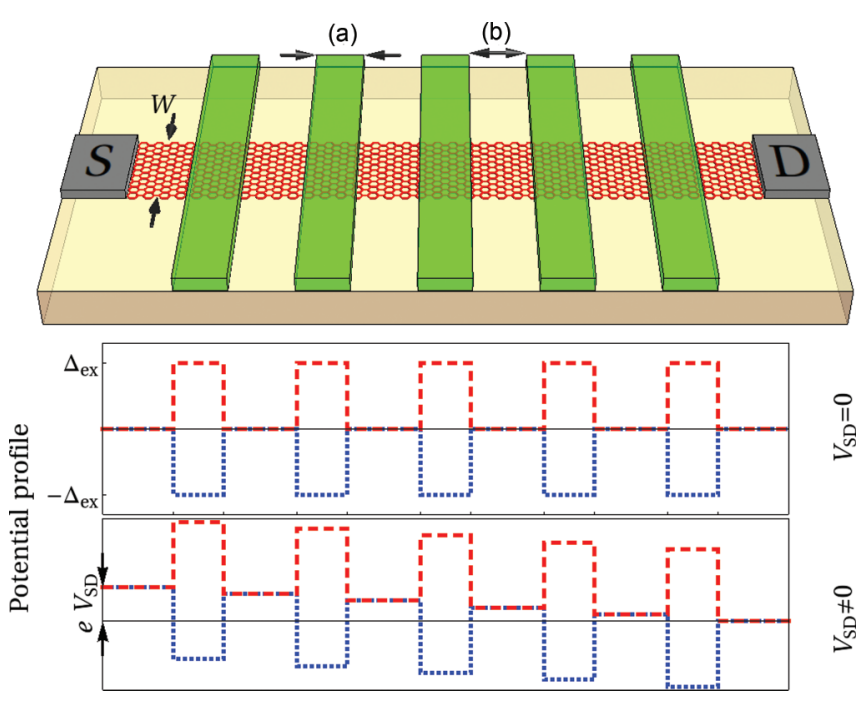

FIG. 1. (Color online) The upper panel shows the GNR connected to source (S) and drain (D) leads, with $N=5$ perpendicular strips of a ferromagnetic insulator (green bars) on top of it. The model potential profiles for spin-up (dashed red lines) and spin-down (dotted blue lines) electrons in the unbiased and biased device are shown in the middle and lower panels, respectively.

hexagon. Contrary to GNRs with zigzag edges, the dispersion relation of the armchair GNR is centered around $k=0$. This is advantageous for tunneling structures because the resonant levels are expected to be broader and less affected by disorder. ${ }^{26}$ We therefore restrict ourselves to the armchair GNRs.

$\mathrm{EuO}$ can be used as the ferromagnetic insulator for the superlattice; this material has been studied in conjunction with graphene both experimentally ${ }^{27,28}$ and theoretically. ${ }^{19}$ The proximity exchange interaction between magnetic ions in the strips and charge carriers in the GNR can be described as an effective Zeeman splitting $\pm \Delta_{\text {ex }}$ of the spin sublevels. ${ }^{19}$ There is still no consensus on the magnitude of the exchange splitting amplitude $\Delta_{\text {ex }}$ in graphene. We use $\Delta_{\text {ex }}=5 \mathrm{meV}$, which lies in the range of values known from the literature $(3-10 \mathrm{meV}) .{ }^{19,29,30}$ We have checked that our results do not change qualitatively if we use a different value of $\Delta_{\mathrm{ex}}$ within the known range.

Because the proximity exchange interaction has the characteristic length scale of one atomic layer, the splitting is induced only in the regions of the GNR which are just below the ferromagnetic strips. Therefore, for the chosen system geometry, a spin-up (spin-down) electron propagating along the sample will be subjected to a potential comprising a periodic set of rectangular barriers (wells), as plotted in the middle panel of Fig. 1. In other words, the array of the ferromagnetic strips creates a spin-dependent superlattice. We note that similar systems manifesting NDR have been studied in Ref. 23, but the superlattice potential was supposed to be induced by electrostatic gates, so all characteristics were spin independent.

\section{A. Tight-binding method}

A simple tight-binding Hamiltonian of a single electron in the $p_{z}$ orbitals of graphene is widely used to model GNRs. For low-energy excitations, i.e., energies close to the Dirac point, hopping can be restricted to the nearest neighbors. Then, the Hamiltonian can be written as

$$
\mathcal{H}=-t \sum_{\langle i, j\rangle}|i\rangle\left\langle j\left|+\sum_{i} \epsilon_{i}\right| i\right\rangle\left\langle i\left|+\sigma \Delta_{\text {ex }} \sum_{i \in \mathcal{L}}\right| i\right\rangle\langle i| .
$$

Here $|i\rangle$ is the ket vector of the atomic orbital of the $i$ th carbon atom, $t=2.8 \mathrm{eV}$ is the hopping between neighboring atoms, the full set of which is denoted as $\langle i, j\rangle$. The on-site energy is the sum of the following two terms: the bias-induced electrostatic potential $\epsilon_{i}$ at the position of the $i$ th atom (see Sec. III B) and the spin-dependent exchange-interaction $\Delta_{\mathrm{ex}}$ due to the ferromagnetic strips, with $\sigma= \pm 1$ for spin-up and spin-down electrons. The exchange-interaction is induced only at the atoms that are in direct contact with the ferromagnetic strips (the full set of them is labeled as $\mathcal{L}$ in the above equation). The on-site energy is sketched, for zero and finite bias, in the middle and lower panels of Fig. 1, respectively.

The wave function in the GNR can be obtained using the quantum transmission boundary method..$^{31,32}$ This is accomplished by assuming semi-infinite leads, whose modes are calculated using an effective transfer-matrix approach. ${ }^{33}$ Then, both the ingoing and outgoing wave functions are computed as linear combinations of propagating plane waves at a given energy, and the corresponding amplitudes determine the spin-dependent transmission probabilities $T_{ \pm}$.

\section{B. Dirac theory}

For not too narrow GNRs, the low-energy excitations can be treated very efficiently within the Dirac approximation. ${ }^{34,35}$ Boundary conditions of GNRs require the wave function to vanish on the (fictitious) sites just outside the GNR, i.e., at $y=0$ and $y=W+a_{0}$, where the $y$ axis is perpendicular to the direction of the GNR and the lower edge of the GNR is located at $y=a_{0} / 2$ [see Fig. 2(a)], where $a_{0}$ is the lattice period along the $y$ direction. In the case of armchair GNRs, this affects both sublattices and the boundary conditions can be fulfilled by a superposition of two states from different valleys with the same energy $E=\hbar v_{\mathrm{F}}\left(k_{\perp}^{2}+k_{\|}^{2}\right)^{1 / 2}$ and equal longitudinal momentum $\hbar \boldsymbol{k}_{\|}$, but with opposite transverse momentum $\pm \hbar \boldsymbol{k}_{\perp}$, measured from the Dirac points. ${ }^{17,36}$ Here $v_{\mathrm{F}}$ is the Fermi velocity in graphene. Note that the effective description given by the Dirac equation holds as long as the $\boldsymbol{k} \cdot \boldsymbol{p}$ approximation remains valid, i.e., for not too narrow GNRs.

Since the valley momenta $\boldsymbol{K}$ and $\boldsymbol{K}^{\prime}$ can be chosen parallel to $\boldsymbol{k}_{\perp}$, the transverse wave function can be written $\phi_{\perp}(y)=$ $\sin \left[\left(K+k_{\perp}\right) y\right]$ where $K=4 \pi / 3 a_{0}$. This function is evaluated on the honeycomb lattice with $y \in \mathbb{N} a_{0} / 2$ and oscillates rapidly. The transverse momentum $\boldsymbol{k}_{\perp}$, however, is small and quantized by the conditions $\phi_{\perp}\left(W+a_{0}\right)=\phi_{\perp}(0)=0$. The allowed values for $k_{\perp}$ are given by $\left(K+k_{\perp n}\right)\left(W+a_{0}\right)=\mathbb{Z} \pi$, and the spectrum reads

$$
E_{n}\left(k_{\|}\right)= \pm \hbar v_{\mathrm{F}} \sqrt{k_{\perp n}^{2}+k_{\|}^{2}} .
$$

Taking into account that $W$ is an integer multiple of $a_{0} / 2$, one finds that the spectrum is gapless if ${ }^{17}$

$$
W=\left(3 n_{1}+1\right) a_{0} / 2, \quad n_{1} \in \mathbb{N} .
$$



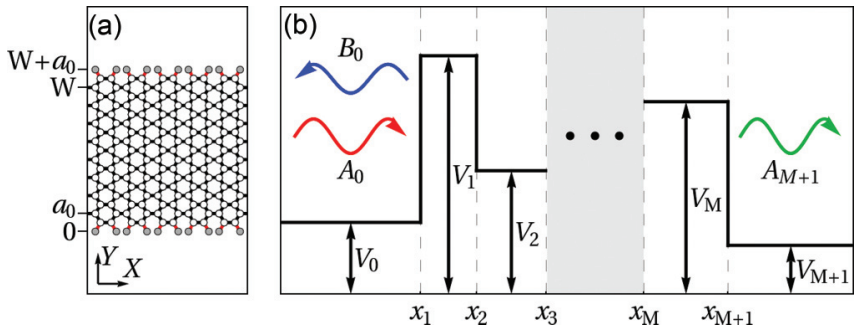

FIG. 2. (Color online) (a) Scheme of a GNR of width $W=8 a_{0}$. The boundary conditions for wave functions can be obtained by adding two rows of atoms (plotted in gray) at $y=0$ and $W+a_{0}$ and setting the wave function to 0 in those points. (b) Transmission across a series of $M$ potential steps. The incident plane wave with amplitude $A_{0}$ splits into a reflected and a transmitted component with amplitudes $B_{0}$ and $A_{M+1}$, respectively.

For asymmetric armchair GNRs, as in Ref. $36, n_{1}$ is even. For symmetric armchair GNRs, $W$ is an integer multiple of $a_{0}$ and $n_{1}$ is odd, such that $W=(3 n-1) a_{0}, n \in \mathbb{N}$ implies a gapless spectrum.

In real samples there are small gaps even in the case (3), which are due to edge effects ${ }^{24,25}$ not included in the simple Dirac ansatz nor the homogeneous tight-binding formulation. In this work we consider symmetric armchair GNRs of width $W=\tilde{n} a_{0}$, where the integer $\tilde{n}$ is different from $3 \mathbb{N}-1$, e.g., $W=40 a_{0}$. In this case, there is a band gap already due to the above reasoning, and the edge effects are negligible. Then, the allowed values of the transverse momentum are

$$
\left|k_{\perp n}\right|=\frac{\pi n}{3\left(W+a_{0}\right)}, \quad n=1,2,4,5,7,8, \ldots,
$$

and the half gap is $E_{0}=E_{1}(0)=\pi \hbar v_{\mathrm{F}} /\left[3\left(W+a_{0}\right)\right]=$ $61.9 \mathrm{meV}$. In the following, we will consider only the lowest transverse momentum $k_{\perp 1}$ and omit the index 1 .

\section{Transfer-matrix description of transmission}

For potentials depending only on the longitudinal coordinate $x$, the transverse momentum $\boldsymbol{k}_{\perp}$ together with the wave function $\phi_{\perp}(y)$ is conserved, and it suffices to solve for the longitudinal wave function $\phi_{\|}(x)$. We consider the transmission across a piecewise constant potential profile, as sketched in Fig. 2(b). The solution of the Dirac equation for each spin $\sigma= \pm 1$ and in each interval of constant potential value $V$ is the superposition of two counterpropagating sublattice pseudospinors

$$
\psi_{\|}(x)=A\left(\begin{array}{c}
e^{-i \theta / 2} \\
-e^{i \theta / 2}
\end{array}\right) e^{i k_{\|} x}+B\left(\begin{array}{c}
e^{+i \theta / 2} \\
-e^{-i \theta / 2}
\end{array}\right) e^{-i k_{\|} x},
$$

with $\tan \theta=k_{\|} / k_{\perp}$ and $k_{\|}=\left[(E-V)^{2} /\left(\hbar v_{\mathrm{F}}\right)^{2}-k_{\perp}^{2}\right]^{1 / 2}$. The solution may be evanescent because Eq. (5) holds also for $|E-V|<\hbar v_{\mathrm{F}}\left|k_{\perp}\right|$, when $k_{\|}$and $\theta$ become imaginary. Then, the general form of the wave function in each slab $j$ with potential $V_{j}$ and momentum $k_{\|}=k_{j}$ is

$$
\left(\begin{array}{cc}
e^{-i \theta_{j} / 2} & e^{i \theta_{j} / 2} \\
-e^{i \theta_{j} / 2} & -e^{-i \theta_{j} / 2}
\end{array}\right)\left(\begin{array}{c}
A_{j}(x) \\
B_{j}(x)
\end{array}\right)=: S_{j}\left(\begin{array}{c}
A_{j}(x) \\
B_{j}(x)
\end{array}\right),
$$

where $A_{j}(x)=A_{j} e^{i k_{j} x}$ and $B_{j}(x)=B_{j} e^{-i k_{j} x}$, such that

$$
\left(\begin{array}{c}
A_{j}\left(x_{j+1}\right) \\
B_{j}\left(x_{j+1}\right)
\end{array}\right)=G_{j}\left(\begin{array}{c}
A_{j}\left(x_{j}\right) \\
B_{j}\left(x_{j}\right)
\end{array}\right)
$$

with $G_{j}=e^{i k_{j}\left(x_{j+1}-x_{j}\right) \sigma_{z}}$. At each junction, $k_{j}$ changes but the wave function remains continuous:

$$
S_{j}\left(\begin{array}{c}
A_{j}\left(x_{j}\right) \\
B_{j}\left(x_{j}\right)
\end{array}\right)=S_{j-1}\left(\begin{array}{c}
A_{j-1}\left(x_{j}\right) \\
B_{j-1}\left(x_{j}\right)
\end{array}\right) .
$$

With the help of Eqs. (7) and (8), one writes down the transfer matrix for the whole system

$$
\left(\begin{array}{c}
A_{M+1} \\
B_{M+1}
\end{array}\right)=S_{M+1}^{-1} \widetilde{G}_{M} \ldots \widetilde{G}_{2} \widetilde{G}_{1} S_{0}\left(\begin{array}{c}
A_{0} \\
B_{0}
\end{array}\right)
$$

with $\widetilde{G}_{j}=S_{j} G_{j} S_{j}^{-1}$.

For the transmission problem depicted in Fig. 2(b), the boundary condition is no incoming electron from the right, $B_{M+1}=0$. The reflection probability at the left is the ratio of reflected to incident current, $R=\left|B_{0}\right|^{2} /\left|A_{0}\right|^{2}$. For the transmission probability one has to take into account that the longitudinal momenta $k_{M+1}$ and $k_{0}$ are different if $V_{0} \neq V_{M+1}$, such that the ratio of transmitted to incident current is $T=$ $\left(\left|A_{M+1}\right|^{2} k_{M+1}\right) /\left(\left|A_{0}\right|^{2} k_{0}\right)$.

\section{Band structure of the unbiased lattice}

The Dirac formalism allows us to analytically study the system in the limit $N \rightarrow \infty$, when the energy regions with high transmission become transmission bands surrounded by insulating bands with $T=0$. For an unbiased lattice with identical barriers of width $a$ and spacing $b$, there are only two different transfer matrices involved, $\widetilde{G}_{a}$ and $\widetilde{G}_{b}$. In the limit $N \rightarrow \infty$, the superlattice eigenfunctions have the Bloch phases $\exp ( \pm i q l)$, that are the eigenvalues of the transfer matrix $\widetilde{G}=\widetilde{G}_{a} \widetilde{G}_{b}$ over one lattice period $l=a+b$. Thus, the dispersion relation $E\left(q, k_{\perp}\right)$ is obtained as $\cos (q l)=\operatorname{Tr}(\widetilde{\mathrm{G}}) / 2$, or again 37,38

$$
\cos q l=\cos k_{a} a \cos k_{b} b+\frac{\cos \theta_{a} \cos \theta_{b}-1}{\sin \theta_{a} \sin \theta_{b}} \sin k_{a} a \sin k_{b} b .
$$

If $|\operatorname{Tr}(\widetilde{\mathrm{G}}) / 2|>1$, there is no propagating solution with realvalued $q$, and $E$ falls into the band gap of the superlattice. In Fig. 3(a), the transmission bands for both spin channels are indicated by the extended bars on the bottom.

\section{E. Spin-polarized current at finite bias}

Because the superlattice potential depends on the carrier spin, the transmission probability $T_{ \pm}$is also spin-dependent. Hereafter, $+(-)$ signs and red (blue) colors in all figures correspond to spin-up (spin-down) electrons, respectively. In order to to calculate the spin-dependent electric currents $I_{ \pm}$ across the sample from the transmission probabilities $T_{ \pm}$, we use the Landauer-Büttiker scattering formalism ${ }^{39}$

$$
I_{ \pm}=\frac{2 e}{h} \int T_{ \pm}\left(E, V_{\mathrm{SD}}\right)\left[f\left(E-\mu_{\mathrm{S}}\right)-f\left(E-\mu_{\mathrm{D}}\right)\right] d E,
$$

where $f(\epsilon)=\left[\exp \left(\epsilon / k_{\mathrm{B}} T\right)+1\right]^{-1}$ is the Fermi-Dirac distribution at temperature $T$. We address the current and its 

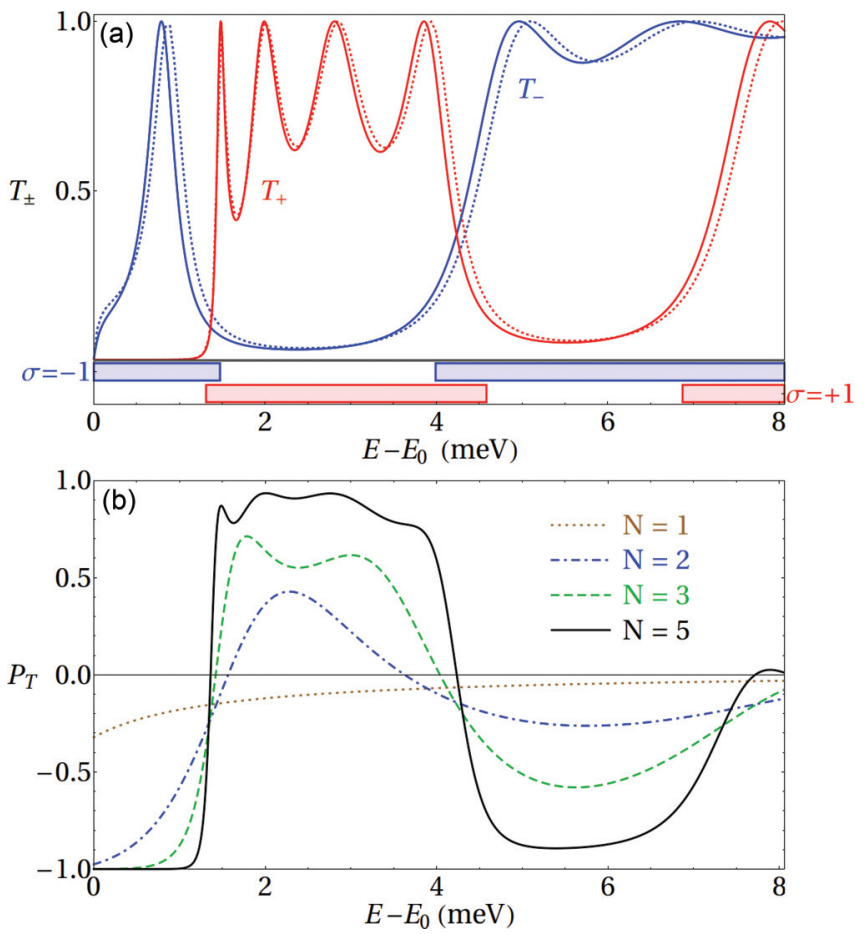

FIG. 3. (Color online) (a) Transmission probabilities $T_{ \pm}$as functions of energy for spin-up (red) and spin-down (blue) electrons. There is a very good agreement between the Dirac approximation (solid lines) and the tight-binding calculation (dotted lines). Horizontal bars in the lower part of the figure indicate the energy bands of the infinite superlattice, obtained from (10). (b) Transmission polarization $P_{T}$ as a function of energy for different numbers $N$ of ferromagnetic strips.

polarization at a finite bias voltage $V_{\mathrm{SD}}$ between source and drain, whose chemical potentials, $\mu_{\mathrm{S}}=\mu+e V_{\mathrm{SD}}$ and $\mu_{\mathrm{D}}=\mu$, have the same offset $\mu$ from the Dirac point. Using $I_{+}$ and $I_{-}$we can calculate the total current $I=I_{+}+I_{-}$through the device, as well as its spin-polarization $P=\left(I_{+}-I_{-}\right) / I$.

\section{RESULTS}

\section{A. Transmission at zero bias}

Figure 3(a) shows the transmission probability through the unbiased sample calculated within the Dirac approximation (solid lines) and the full tight-binding model (dotted lines). Already for the relatively small number $N=5$ of strips, regions of high transmission coincide quite well with the bands of the infinite superlattice introduced in Sec. II D (and indicated by the horizontal bars at the bottom of the figure). The origin of the energy for each curve is set to the lowest subband bottom energy $E_{0}$ calculated within the corresponding model. The figure demonstrates very good agreement between the two approaches. The Dirac approximation overestimates slightly the value of $E_{0}$ (by $0.3 \%$ ) but is accurate enough for our purposes. Unless stated otherwise, in the following, we use the Dirac approximation since it demands less computational resources.

The transmission is spin-dependent, which manifests itself clearly in the transmission polarization, defined as $P_{T}=$ $\left(T_{+}-T_{-}\right) /\left(T_{+}+T_{-}\right)$and shown in Fig. 3(b). As the number of strips is increased, the transmission probability at energies outside the transmission bands vanishes rapidly, thus leading to an enhanced polarization. For $N \geqslant 3$, the transmission polarization noticeably changes within narrow energy intervals. Such abrupt polarization switching can be expected only if the overlap between transmission bands corresponding to different spins is small, as seen in Fig. 3(a). The overlap is determined by different factors: the splitting $\Delta_{\mathrm{ex}}$ and the geometrical parameters $a$ and $b$, which should be chosen carefully in order to observe a pronounced switching and filtering effect in a real device. Such a choice can be made, for example, by analyzing the band structure of the infinite lattice within the Dirac approximation given by (10).

\section{B. Spin-polarized current at finite bias}

As depicted in the lower panel of Fig. 1, we assume the bias voltage to drop along the sample in a roughly Ohmic manner. For simplicity, we assume that the voltage drops occur at the edges of the EuO strips only, resulting in a piecewise constant potential profile as shown in the lower panel of Fig. 1. Such a model potential allows us to use the efficient Dirac transfer matrix method discussed in Sec. II C. There may be additional voltage drops at the source and drain contacts, which are just outside the middle and lower panels of Fig. 1. With the term bias voltage $V_{\mathrm{SD}}$, we refer only to the voltage drop across the GNR. The exact potential profile can in principle be obtained from a self-consistent electrostatic potential calculation, but that would go beyond the scope of this work. We note that, since the desired potential profile $\epsilon_{i}$ is spin independent, it can always be adjusted via gate voltages. In the Appendix we demonstrate that the spin-dependent transmission does not depend crucially on the details of the biased potential profile.

The bias results in a distortion of the transmission bands: the bands shift, quench, and finally disappear as the voltage increases, as is seen in Figs. 4(a) and 4(b) compared to Fig. 3(a). The polarized currents $I_{ \pm}$as functions of $V_{\mathrm{SD}}$ are plotted in Figs. 4(c) and 4(d). The spin-dependent transmission bands and their distortion due to the bias lead to NDR regions at different values of the bias voltage for different spins. For spin down, the NDR occurs at a lower bias and the negative slope of the current-voltage curve is particularly steep, which is due to the fact that the first transmission peak remains very sharp until it disappears [Figs. 4(a) and 4(b)]. The lowest spin-up transmission band gets washed out before it disappears at a higher bias, resulting in the less pronounced NDR.

We further address the total current $I$ through the device, as well as its spin-polarization $P$. Figures 4(e) and 4(f) show that the total current $I$ also manifests NDR for two different biases, corresponding to the NDR regions of $I_{-}$and $I_{+}$. The current polarization shows an initial range with negative values followed by a second region dominated by the spin-up current. As the bias increases further, the polarization decays and finally vanishes. Note that the current is highly polarized for certain biases, which proves that the device can operate as a spin filter. On the other hand, because the characteristics $I_{+}\left(V_{\mathrm{SD}}\right)$ and $I_{-}\left(V_{\mathrm{SD}}\right)$ are very different, if the source feeds partially polarized electrons, the total current through the device would depend on the degree of the electron polarization. The latter opens a possibility to determine the polarization of 

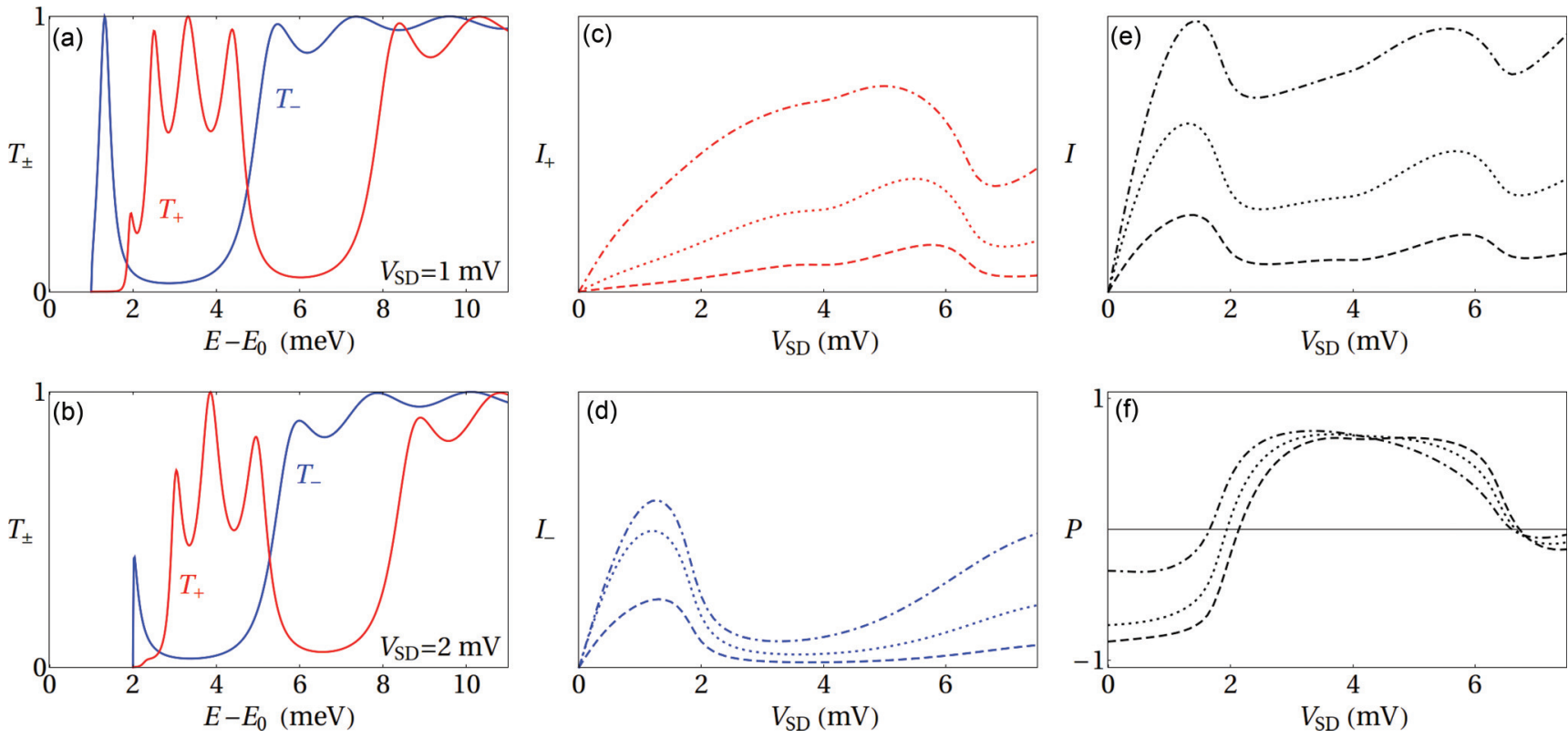

FIG. 4. (Color online) Panels (a) and (b) show that the transmission bands for both spins at finite bias $V_{\mathrm{SD}}$ are shifted, quenched and distorted compared to the unbiased case [Fig. 3(a)]. Panels (c) and (d) display the spin-polarized currents $I_{ \pm}$as functions of the bias $V_{\mathrm{SD}}$, for $T=4 \mathrm{~K}$ and different values of the chemical potential: $\mu-E_{0}=0 \mathrm{meV}$ (dashed), $0.5 \mathrm{meV}$ (dotted), and $1.0 \mathrm{meV}$ (dash-dotted) lines. Finally, panels (e) and (f) show the total current $I=I_{+}+I_{-}$and the current polarization $P=\left(I_{+}-I_{-}\right) / I$ for the same parameters. All the intensity graphs use the same arbitrary scale.

a current by purely electrical measurements, which is a very promising application.

We have considered an ideal device with perfect rectangular GNR and strips, while different imperfections and perturbations can introduce disorder into the system and affect the electric current and its polarization. ${ }^{38}$ There are different possible sources of disorder, for example, charged impurities in the substrate or defects of the device fabrication. The former results in an additional smooth electrostatic potential and can hardly deteriorate the transmission through the device to a large extent. However, the effect of the latter on the transport properties can be stronger. To estimate the possible impact of the fabrication imperfections on the predicted effects, we considered disordered superlattices with randomly varying strip widths and spacings, up to $20 \%$. Our calculations (not shown here) demonstrated that the transmission bands are affected by the disorder to a comparable degree for both spin up and spin down electrons, which suggests that a moderate disorder would not seriously deteriorate transport and polarization properties of the device. The current magnitude remains almost the same, and the NDR turns out to be robust under the effects of disorder as well.

\section{CONCLUSIONS}

In summary, we propose a graphene-based device comprising a GNR and a regular array of ferromagnetic strips on top of it. The ferromagnets induce a proximity exchange splitting of the electronic states in the GNR and create a spin-dependent superlattice. We have shown that the electric current through the device can be highly polarized. Thus, the device can operate as a spin filter. Alternatively, it can be used to obtain the polarization degree of the source electrons by purely electrical measurements. Moreover, the two polarized components of the current manifest nonmonotonic dependencies on the bias voltage. In particular, for both spins, the current-voltage characteristics present regions with negative differential resistance for the bias in the range of a few millivolts. The device operates therefore as a low-voltage Esaki diode for spin-polarized currents.

An important advantage of the superlattice induced by ferromagnets is that the exchange interaction is very shortranged; its characteristic length scale is on the order of one monolayer. Unlike the long-range electrostatic gate potentials which can interfere with each other, setting a practical lower limit for the interdevice spacing, the exchange-interaction induced potential profiles are very abrupt. Therefore, heterostructures created by ferromagnets allow for very close packing of circuits and, consequently, considerably higher device densities.

Finally, we note that in a spintronic device the degree of freedom that carries information is the polarization of the current rather than its magnitude. We have shown that the current polarization is also a nonmonotonic function of the bias voltage, suggesting that the superlattice can be used as a Esaki spin diode. This opens a possibility to design a whole new class of spintronic circuits such as spin oscillators, amplifiers, and triggers.

\section{ACKNOWLEDGMENTS}

Work in Madrid was supported by the MICINN (Project No. MAT2010-17180). Research of C.G. was supported by the PICATA postdoctoral fellowship from the Moncloa Campus of International Excellence (UCM-UPM). P.A.O. acknowledges financial support from the FONDECYT (Grant No. 1100560). 
The Centre for Quantum Technologies is a Research Centre of Excellence funded by the Ministry of Education and the National Research Foundation of Singapore.

\section{APPENDIX: ROBUSTNESS AGAINST DETAILS OF THE POTENTIAL PROFILE}

To estimate the accuracy of the transmissions $T_{ \pm}$obtained using the piecewise constant potential of the main text, we compared them with those calculated using a different potential profile, where a linear gradient $V_{\mathrm{SD}} /(5 a+6 b)$ is added to the spin-dependent superlattice potential of the middle panel of Fig. 1. Results obtained with the tight-binding method are shown in Fig. 5. There are only slight deviations, which proves that the details of the potential are not important for our findings and that the piecewise constant potential is a very good approximation.

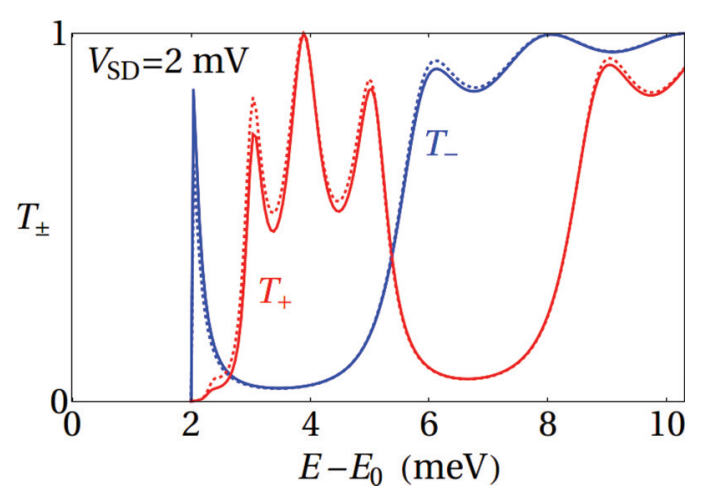

FIG. 5. (Color online) Transmissions $T_{ \pm}$for both spins at finite bias $V_{\mathrm{SD}}=2 \mathrm{mV}$, assuming that the voltage drop occurs at the edges of the EuO strips (solid) or linearly along the sample (dashed). Here, we use the tight-binding model, which can be compared with the Dirac approximation on Fig. 4(b).
${ }^{1}$ L. Esaki, Phys. Rev. 109, 603 (1958).

${ }^{2}$ L. Esaki, Rev. Mod. Phys. 46, 237 (1974).

${ }^{3}$ S. Sze and K. Ng, Physics of Semiconductor Devices (John Wiley \& Sons, New York, 2006).

${ }^{4}$ A. V. Malyshev, Phys. Rev. Lett. 98, 096801 (2007).

${ }^{5}$ R. Tsu and L. Esaki, Appl. Phys. Lett. 22, 562 (1973).

${ }^{6}$ R. Kümmel, U. Gunsenheimer, and R. Nicolsky, Phys. Rev. B 42, 3992 (1990).

${ }^{7}$ F. Léonard and J. Tersoff, Phys. Rev. Lett. 85, 4767 (2000).

${ }^{8}$ P. Bedrossian, D. M. Chen, K. Mortensen, and J. A. Golovchenko, Nature (London) 342, 258 (1989).

${ }^{9}$ K. S. Novoselov, A. K. Geim, S. V. Morozov, D. Jiang, Y. Zhang, S. V. Dubonos, I. V. Grigorieva, and A. A. Firsov, Science 306, 666 (2004).

${ }^{10}$ C. L. Kane and E. J. Mele, Phys. Rev. Lett. 95, 226801 (2005).

${ }^{11} \mathrm{~N}$. Tombros, C. Jozsa, M. Popinciuc, H. T. Jonkman, and B. J. van Wees, Nature (London) 448, 571 (2007).

${ }^{12}$ W. Han, J.-R. Chen, D. Wang, K. M. McCreary, H. Wen, A. G. Swartz, J. Shi, and R. K. Kawakami, Nano Lett. 12, 3443 (2012).

${ }^{13}$ P. J. Zomer, M. H. D. Guimarães, N. Tombros, and B. J. van Wees, Phys. Rev. B 86, 161416 (2012).

${ }^{14}$ M. B. Lundeberg, R. Yang, J. Renard, and J. A. Folk, Phys. Rev. Lett. 110, 156601 (2013).

${ }^{15}$ A. V. Rozhkov, G. Giavaras, Y. P. Bliokh, V. Freilikher, and F. Nori, Phys. Rep. 503, 77 (2011).

${ }^{16} \mathrm{~J}$. Munárriz, F. Domínguez-Adame, P. A. Orellana, and A. V. Malyshev, Nanotechnology 23, 205202 (2012).

${ }^{17}$ K. Wakabayashi, Y. Takane, M. Yamamoto, and M. Sigrist, New J. Phys. 11, 095016 (2009).

${ }^{18}$ T.-T. Wu, X.-F. Wang, M.-X. Zhai, H. Liu, L. Zhou, and Y.-J. Jiang, Appl. Phys. Lett. 100, 052112 (2012).

${ }^{19}$ H. Haugen, D. Huertas-Hernando, and A. Brataas, Phys. Rev. B 77, 115406 (2008).

${ }^{20}$ X.-X. Yu, Y.-E. Xie, Y. T. Ou, and Y.-P. Chen, Chin. Phys. B 21, 107202 (2012).
${ }^{21}$ Z. P. Niu, F. X. Li, B. G. Wang, L. Sheng, and D. Y. Xing, Eur. Phys. J. B 66, 245 (2008).

${ }^{22}$ E. Faizabadi, M. Esmaeilzadeh, and F. Sattari, Eur. Phys. J. B 85, 198 (2012).

${ }^{23}$ G. J. Ferreira, M. N. Leuenberger, D. Loss, and J. C. Egues, Phys. Rev. B 84, 125453 (2011).

${ }^{24}$ M. Y. Han, B. Özyilmaz, Y. Zhang, and P. Kim, Phys. Rev. Lett. 98, 206805 (2007)

${ }^{25}$ Y.-W. Son, M. L. Cohen, and S. G. Louie, Phys. Rev. Lett. 97, 216803 (2006)

${ }^{26}$ J. Munárriz, F. Domínguez-Adame, and A. V. Malyshev, Nanotechnology 22, 365201 (2011).

${ }^{27}$ D. F. Förster, Ph.D. thesis, Universität zu Köln, 2011.

${ }^{28}$ A. G. Swartz, P. M. Odenthal, Y. Hao, R. S. Ruoff, and R. K. Kawakami, ACS Nano 6, 10063 (2012).

${ }^{29}$ J. Zou, G. Jin, and Y.-Q. Ma, J. Phys.: Condens. Matter 21, 126001 (2009).

${ }^{30}$ Y. Gu, Y. H. Yang, J. Wang, and K. S. Chan, J. Appl. Phys. 105, 103711 (2009).

${ }^{31}$ C. S. Lent and D. J. Kirkner, J. Appl. Phys. 67, 6353 (1990).

${ }^{32}$ D. Z.-Y. Ting, E. T. Yu, and T. C. McGill, Phys. Rev. B 45, 3583 (1992).

${ }^{33}$ J. Schelter, D. Bohr, and B. Trauzettel, Phys. Rev. B 81, 195441 (2010).

${ }^{34}$ P. R. Wallace, Phys. Rev. 71, 622 (1947).

${ }^{35}$ A. H. Castro Neto, F. Guinea, N. M. R. Peres, K. S. Novoselov, and A. K. Geim, Rev. Mod. Phys. 81, 109 (2009).

${ }^{36}$ L. Brey and H. A. Fertig, Phys. Rev. B 73, 235411 (2006).

${ }^{37}$ M. Barbier, P. Vasilopoulos, and F. M. Peeters, Phys. Rev. B 81, 075438 (2010).

${ }^{38}$ Q. Zhao, J. Gong, and C. A. Müller, Phys. Rev. B 85, 104201 (2012).

${ }^{39}$ M. Büttiker, Y. Imry, R. Landauer, and S. Pinhas, Phys. Rev. B 31, 6207 (1985). 